\title{
Predicting type 2 diabetes mellitus: a comparison between the FINDRISC score and the metabolic syndrome
}

\author{
Abraham S. Meijnikman ${ }^{*}{ }^{\dagger}$, Christophe E. M. De Block ${ }^{\dagger}$, An Verrijken, Ilse Mertens and Luc F. Van Gaal
}

\begin{abstract}
Background: The aim of this study to compare the diagnostic accuracy of the metabolic syndrome (MetS) with the FINDRISC score to screen for type 2 diabetes mellitus T2DM in an overweight/obese population.

Methods: Subjects 18 years or older visiting the obesity clinic of the Antwerp University Hospital were consecutively recruited between 2012 and 2014. Every patient underwent a standard metabolic work-up including a clinical examination with anthropometry. Glucose status was tested using OGTT and Hba1c. FINDRISC questionnaire and MetS were examined.

Results: Of 651 subjects, 50.4\% were diagnosed with prediabetes, whereas $11.1 \%$ was diagnosed with T2DM. FINDRISC score increased with worsening of glucose status $11 \pm 3,13 \pm 4$ and $15 \pm 5$ in respectively, subjects without T2DM, prediabetes and T2DM. 312 subjects had the MetS. The aROC of the FINDRISC to identify subjects with T2DM was 0.76 (95\% Cl 0.72-0.82), sensitivity was $64 \%$ and specificity was $63 \%$ with 13 as cutoff point. Adding FPG or HbA1c to FINDRISC, the aROC increased significantly to 0.91 (95\% Cl $0.88-0.95)$ and $0.93(95 \% \mathrm{Cl} 0.90-0.97)$, respectively $(p<0.001)$. The aROC of the MetS to identify subjects with diabetes was $0.72(95 \% \mathrm{Cl} 0.65-0.78)$, sensitivity was $75 \%$ and specificity was $55 \%$. The aROC of the FINDRISC + HbA1c was significantly higher than the MetS for predicting T2DM $(p<0.001)$.

Conclusion: Prediction of type 2 diabetes is important for timely intervention and to avoid chronic complications associated with the disease. Our findings suggest, that it may be of good clinical practice to use the FINDRISC score $+\mathrm{HbA} 1 \mathrm{c}$ in a two-step screening model for diabetes rather than using the metabolic syndrome.
\end{abstract}

Keywords: Type 2 diabetes mellitus, Prediabetes, Screening, Metabolic syndrome, FINDRISC, Obesity

\section{Background}

According to the International Diabetes Federation, it is estimated that every $6 \mathrm{~s}$ one person dies from diabetes and more than 600 billion USD is spent on diabetes related healthcare [1]. Despite several prevention programs and attempts to create global awareness of the risks of type 2 diabetes, the prevalence is expected to increase to 642 million in 2040 [1]. Due to the human

\footnotetext{
*Correspondence: s.meijnikman@gmail.com; luc.vangaal@uza.be; luc.van.gaal@uza.be

${ }^{\dagger}$ Abraham S. Meijnikman and Christophe E. M. De Block contributed equally to this work

Department of Endocrinology, Diabetology \& Metabolism, Faculty of Medicine, Antwerp University Hospital and University of Antwerp, Wilrijkstraat 10, 2650 Edegem, Belgium
}

and economical burden of the disease and its complications, there is a tremendous need for a cost efficient, accurate and feasible screening tool for type 2 diabetes. In the majority of the subjects with prediabetes or type 2 diabetes, a set of risk factors commonly appears together, which is now known as the metabolic syndrome. The metabolic syndrome, which is a cluster of insulin resistance and disturbed glucose metabolism, overweight and abdominal fat distribution, dyslipidemia and high blood pressure, is an important determinant of cardiovascular risk [2]. It is estimated that about $25 \%$ of the world adult population has the metabolic syndrome [3] In addition, subjects with the metabolic syndrome have a 5 times higher risk of developing type 2 diabetes [4-7]. 
However, before the diagnosis of the metabolic syndrome can be established, five clinical and biochemical parameters need to be assessed, making it one of the most labor intensive and cost expensive prediction tools for type 2 diabetes mellitus [8].

The Finnish Diabetes Risk Score (FINDRISC) is another commonly used and validated non-invasive diabetes risk score worldwide and has proven its value in the national type 2 diabetes prevention program in Finland [9]. The risk score consists of eight non-invasive clinical characteristics such as age, body mass index (BMI), waist circumference, daily physical activity, eating habits, blood pressure or antihypertensive medication, a history of high blood glucose and family history of diabetes.

However, to the best of our knowledge, the discriminatory accuracy of the metabolic syndrome versus the FINDRISC score has never been tested before. Therefore, we compared the metabolic syndrome to the FINDRISC score as a screening tool for type 2 diabetes mellitus in a population of apparently healthy overweight and obese subjects without a prior diagnosis of diabetes, considered as an 'at risk' population.

\section{Methods}

Subjects 18 years or older visiting the obesity clinic of the Antwerp University Hospital (a tertiary referral facility) for a problem of overweight (BMI $\geq 25-29.9 \mathrm{~kg} / \mathrm{m}^{2}$ ) or obesity (BMI $\geq 30 \mathrm{~kg} / \mathrm{m}^{2}$ ) were consecutively recruited between 2012 and 2014, after written informed consent. Subjects with a history of diabetes or those already taking anti-diabetic medication and non-Caucasian subjects were excluded.

Every patient underwent a standard metabolic workup including a clinical examination with anthropometry. All measurements were performed in the morning, with patients in fasting conditions and undressed. Height was measured to the nearest $0.5 \mathrm{~cm}$ using a wall-mounted stadiometer, and body weight was measured using a digital scale to the nearest $0.2 \mathrm{~kg}$ with subjects in their underwear and without shoes. Body mass index (BMI) was calculated as weight in kilograms over height in square meters. Waist circumference was measured at the midlevel between the lower rib margin and the iliac crest. Visceral abdominal adipose tissue (VAT) and subcutaneous abdominal adipose tissue (SAT) were determined by a 64-slice computerized tomography $(\mathrm{CT})$ scan at the L4-L5 level (slice thickness $0.6 \mathrm{~mm}$ ), according to the technique described by Van der Kooy and Seidell [10] and Kvist [11]. First, the total area of abdominal tissue was measured at -190 to $330 \mathrm{HU}$. Subsequently, the surface area of different fat compartments was assessed using a dedicated software (AW VolumeShare 2). The total adipose tissue area was calculated in the area limited by the skin including the epidermis. Visceral adipose tissue was calculated in the area within the fascia transversalis. The difference between the total fat tissue and the visceral fat was equivalent to the subcutaneous fat tissue.

Fasting and a 2-h oral glucose tolerance test, using 75-g glucose, was performed, and insulin and c-peptide levels were analyzed. Insulin resistance and beta cell function was estimated using homeostasis model assessment (HOMA) as described by Matthews et al. [12]. Insulin resistance (HOMA-IR) was calculated as [insulin $(\mathrm{mU} / \mathrm{l}) \times \operatorname{glucose}(\mathrm{mmol} / \mathrm{l})] / 22.5$, with one as reference value for normal insulin sensitivity. Beta cell function (HOMA-B) was calculated as $20 \times$ (insulin $(\mathrm{mU} / \mathrm{l}) /(\mathrm{glu}-$ cose $(\mathrm{mmol} / \mathrm{l})-3.5)$.

\section{Diabetes and pre-diabetes definitions}

The American Diabetes Association classification for the diagnosis of prediabetes and diabetes was used [13]. Subjects who had a fasting glucose (FPG) $\geq 126 \mathrm{mg} / \mathrm{dl}$ and/or a HbA1c $\geq 6.5 \%$ and/or a 2 -h plasma glucose $\geq 200 \mathrm{mg} /$ $\mathrm{dl}$ were classified as de novo diabetes. Subjects with an impaired fasting glucose (IFG) (100-125 mg/dl) or/and an impaired glucose tolerance (IGT) (140-199 mg/dl) or/and a HbA1c between 5.7 and $6.4 \%$ were classified as being pre-diabetic.

\section{Metabolic syndrome criteria}

The metabolic syndrome was evaluated according to the National Cholesterol Education Program Adult Treatment Panel III (NCEP-ATP III) [8], subjects were classified as having the metabolic syndrome if $\geq$ three of the following criteria were met: (1) abdominal obesity (waist circumference $\geq 102 \mathrm{~cm}$ in men and $\geq 88 \mathrm{~cm}$ in women), (2) hypertriglyceridemia ( $\geq 150 \mathrm{mg} / \mathrm{dl}$ ), (3) low HDL cholesterol $(<40 \mathrm{mg} / \mathrm{dl}$ in men and $<50 \mathrm{mg} / \mathrm{dl}$ in women), (4) high blood pressure ( $\geq 130 / 85 \mathrm{mmHg}$ ) or on anti-hypertensive medication, (5) high fasting plasma glucose $(\geq 100 \mathrm{mg} / \mathrm{dl})$.

\section{Laboratory measurements}

HbA1C was determined by high performance liquid chromatography (Adams ${ }^{\mathrm{TM}}$ A1c HA-8160, ArkrayMenarini instrument, Zaventem, Belgium; reference range: $4.8-6.0 \%$ ). Plasma levels of glucose, creatinin, total cholesterol and triglycerides were measured on Vitros 750 XRC (Ortho Clinical Diagnostics, Johnson \& Johnson, Buckinghamshire, UK). HDL-C was measured on Hitachi 912 (Roche Diagnostics, Mannheim, Germany). Insulin levels were measured with the Medgenic two-site IRMA assay (BioSource, Belgium). Microalbuminuria was measured with nephelometry using a Behring Nephelometer II (Siemens Healthcare Diagnostics Products, Marburg, Germany). 


\section{Statistical methods}

SPSS version 23 was used for statistical analyses. Data are expressed as mean \pm SD for normally distributed variables or as median (interquartile range) when distribution is skewed. The normal distribution of continuous variables was assessed with the Kolmogorov-Smirnov method. Comparison of population characteristics was performed with an independent samples $t$ test or a Mann-Whitney U test, depending on normality. Variables, which were not normally distributed, were log transformed. The receiver-operating characteristic (ROC) curves were constructed to show the relationship between the sensitivity and specificity of the FINDRISC score for identifying subjects with type 2 diabetes. The area under the receiver-operating curve (aROC) was used to evaluate the discriminatory accuracy of the FINDRISC to identify prediabetes and diabetes subjects. An aROC of 1.0 indicates a perfect test with no false positive rate and no false negative rate, an aROC of 0.5 indicates that the test performed not better than chance. The cutoff points to identify prediabetes and diabetes were determined by the point with the shortest distance to the upper left corner in the ROC curve, which was calculated as the square root of [(1-sensitivity) [2] + (1-specificity) [2] ]. The same statistical analysis was performed to evaluate discriminatory accuracy of the metabolic syndrome to identify subjects with type 2 diabetes mellitus.

\section{Results}

In total, 651 (Male/female: 193/458) overweight and obese subjects, with an average age of $43 \pm 13$ year were examined. Baseline characteristics are presented in Table 1 . Exactly 187 subjects were already on anti-hypertensive medication and 76 were on lipid lowering medication (either statin or fibrate).

According to the American Diabetes Association diagnostic criteria, 72 patients were newly diagnosed with type 2 diabetes mellitus, 328 were classified with prediabetes and 251 were considered non-diabetic. In total, 312 subjects met the diagnostic criteria for metabolic syndrome using the NCEP-ATP III criteria. The FINDRISC score increased with worsening of glucose tolerance. In subjects without type 2 diabetes, the FINDRISC score was $11 \pm 3$, in subjects with prediabetes $13 \pm 4$ and in subjects with type 2 diabetes mellitus $15 \pm 5$. The proportion of subjects with the metabolic syndrome also increased with worsening of the glucose tolerance, $32 \%$ in subjects without diabetes, $54 \%$ in subjects with prediabetes and $75 \%$ in subjects with de novo diagnosed diabetes according to the NCEP-ATP III criteria. Similar results were observed when using other criteria for the metabolic syndrome, such as the World Health Organisation criteria and the Harmonized criteria.
Table 1 Baseline characteristics of 651 overweight or obese subjects without a history of diabetes

\begin{tabular}{|c|c|c|c|c|}
\hline & All & Men & Women & $\mathbf{P}$ \\
\hline $\begin{array}{l}\text { Number of } \\
\text { cases }\end{array}$ & 651 & 193 & 458 & \\
\hline Age (years) & $43 \pm 13$ & $46 \pm 12$ & $42 \pm 13$ & 0.03 \\
\hline Weight (kg) & $109.3 \pm 21.2$ & $124.5 \pm 22.1$ & $102.4 \pm 17.2$ & $<0.01$ \\
\hline BMI $\left(\mathrm{kg} / \mathrm{m}^{2}\right)$ & $38.2 \pm 6.1$ & $39.0 \pm 6.4$ & $37.9 \pm 5.9$ & 0.02 \\
\hline $\begin{array}{l}\text { Waist circum- } \\
\text { ference }(\mathrm{cm})\end{array}$ & $116.2 \pm 14.8$ & $126.3 \pm 12.6$ & $112 \pm 13$ & $<0.01$ \\
\hline $\begin{array}{r}\text { Systolic BP } \\
(\mathrm{mmHg})\end{array}$ & $129 \pm 15$ & $133 \pm 14$ & $127 \pm 15$ & 0.02 \\
\hline $\begin{array}{l}\text { Diastolic BP } \\
\quad(\mathrm{mmHg})\end{array}$ & $76 \pm 11$ & $80 \pm 11$ & $75 \pm 10$ & 0.03 \\
\hline $\begin{array}{l}\text { Triglycerides } \\
\text { (mg/dl) }\end{array}$ & $132(97-185)$ & $163(114-220)$ & $125(91-170)$ & $<0.01$ \\
\hline $\begin{array}{l}\text { Total choles- } \\
\text { terol (mg/dl) }\end{array}$ & $198(175-227)$ & $189(167-219)$ & $203(177-230)$ & $<0.01$ \\
\hline $\begin{array}{l}\text { HDL choles- } \\
\text { terol (mg/dl) }\end{array}$ & $49(40-60)$ & $42(35-50)$ & $52(44-63)$ & $<0.01$ \\
\hline $\begin{array}{l}\text { CT visceral fat } \\
\left(\mathrm{cm}^{2}\right)\end{array}$ & $183(131-249)$ & $249(194-315)$ & 157 (113-209) & $<0.01$ \\
\hline $\begin{array}{c}\text { CT subcutane- } \\
\text { ous fat }\left(\mathrm{cm}^{2}\right)\end{array}$ & $593 \pm 145$ & $562 \pm 167$ & $605 \pm 132$ & $<0.01$ \\
\hline CT total fat $\left(\mathrm{cm}^{2}\right)$ & $789 \pm 173$ & $820 \pm 189$ & $775 \pm 164$ & $<0.01$ \\
\hline $\begin{array}{l}\text { Fasting glu- } \\
\text { cose }(\mathrm{mg} / \mathrm{dl})\end{array}$ & $86(80-95)$ & $92(84-101)$ & $84(79-92)$ & 0.01 \\
\hline $\begin{array}{l}\text { 2-h OGTT glu- } \\
\text { cose (mg/dl) }\end{array}$ & $140(118-167)$ & $147(125-185)$ & $138(117-161)$ & $<0.01$ \\
\hline $\mathrm{HbA1c}(\%)$ & $5.5(5.3-5.8)$ & $5.6(5.4-6.0)$ & $5.4(5.2-5.7)$ & 0.03 \\
\hline $\begin{array}{l}\mathrm{HbAlc}(\mathrm{mmol} / \\
\mathrm{mol})\end{array}$ & $37(34-40)$ & $38(36-41)$ & $36(33-37)$ & 0.03 \\
\hline FINDRISC score & $12 \pm 4$ & $13 \pm 4$ & $12 \pm 4$ & 0.54 \\
\hline HOMA-S & $3.3(2.1-5.4)$ & $4.5(2.9-7.0)$ & $3.0(1.8-4.6)$ & $<0.01$ \\
\hline HOMA-B (\%) & $243(153-375)$ & $241(153-361)$ & $247(157-380)$ & 0.65 \\
\hline $\begin{array}{l}\text { Microalbumi- } \\
\text { nuria ( } \mu \mathrm{g} / \\
\text { min) }\end{array}$ & $8.0(5.9-13.0)$ & $9.0(7.0-16.0)$ & $8.0(5.0-12.0)$ & 0.01 \\
\hline
\end{tabular}

Data are expressed as mean \pm standard deviation or median (range)

$B M I$ body mass index, $B P$ blood pressure, $H D L$ high density lipoprotein, OGTT oral glucose tolerance test, $H O M A-S$ homeostasis model assessment of insulin resistance, $H O M A-B$ homeostasis model assessment of beta-cell function

The discriminatory accuracy of the FINDRISC for identifying subjects with diabetes, assessed by the aROC was 0.76 (95\% CI 0.72-0.82). The cutoff point of the FINDRISC score for detecting diabetes with the shortest distance to the upper left corner of the ROC plot proved to be a FINDRISC score of 13 with a sensitivity of $64 \%$ and specificity of $63 \%$ as previously reported by our group [8]. The positive predictive value was $18 \%$ and the negative predictive value was $93 \%$. When adding FPG or HbA1c to the original FINDRISC score to identify diabetes, the aROC significantly increased to $0.91(95 \%$ CI $0.88-0.95)$ and $0.93(95 \%$ CI $0.90-0.97)$, respectively $(\mathrm{p}<0.001)$. 
Of the 312 subjects who were diagnosed with metabolic syndrome, 151 had a FINDRISC score $>13$ when using the NCEP-ATP III criteria.

The aROC of the FINDRISC to identify subjects with the metabolic syndrome according to the NCEP-ATP III criteria was 0.69 (95\% CI 0.64-0.75). The discriminatory accuracy of the metabolic syndrome assessed by the aROC for identifying subjects with diabetes was 0.72 (95\% CI $0.65-0.78$ ) with a sensitivity of $75 \%$ and specificity of $55 \%$. The positive predictive value was $17 \%$ and the negative predictive value was $95 \%$.

The aROC of the original FINDRISC score did not differ significantly to the aROC of the metabolic syndrome to identify subjects with diabetes. However, by adding FPG or HbA1c, the discriminatory accuracy of the model did differ significantly compared to the metabolic syndrome $(\mathrm{p}<0.001)$.

Characteristics of subjects with a FINDRISC score $<13$ corresponds with the characteristics of the subjects without the metabolic syndrome and the same applies for subjects with a FINDRISC score $\geq 13$ and subjects with the metabolic syndrome (see Table 2).

\section{Correlation analysis}

Univariate analyses showed significant correlations between the FINDRISC score and HOMA-IR $(\mathrm{r}=0.24$, $\mathrm{p}<0.001)$, HOMA-B $(\mathrm{r}=-0.09, \mathrm{p}=0.021)$, HbA1c $(\mathrm{r}=0.35, \mathrm{p}<0.001)$, FPG $(\mathrm{r}=0.34 \mathrm{p}<0.001), 2$ h OGTT glucose $(\mathrm{r}=0.34, \mathrm{p}<0.001)$, VAT $(\mathrm{r}=0.34, \mathrm{p}<0.001)$, VAT/SAT ratio $(\mathrm{r}=0.39, \mathrm{p}<0.001)$ and microalbuminuria $(\mathrm{r}=0.12, \mathrm{p}=0.002)$.

The metabolic syndrome was significantly correlated with the FINDRISC score $(\mathrm{r}=0.24, \mathrm{p}<0.001)$, HOMAIR $(\mathrm{r}=0.31, \mathrm{p}<0.001)$, VAT $(\mathrm{r}=0.33, \mathrm{p}<0.001)$, VAT/SAT ratio $(r=0.31, p<0.001)$, HbA1c $(r=0.22$, $\mathrm{p}<0.001)$, FPG $(\mathrm{r}=0.27, \mathrm{p}<0.001), 2$-h OGTT glucose $(\mathrm{r}=0.28, \mathrm{p}<0.001)$ and microalbuminuria $(\mathrm{r}=0.18$, $\mathrm{p}<0.001)$.

\section{Discussion}

Prediction of type 2 diabetes is important for timely intervention and to avoid chronic complications associated with the disease. To the best of our knowledge, this is the first study comparing the discriminatory accuracy of the metabolic syndrome versus the FINDRISC score

Table 2 Comparison between subjects with a FINDRISC score $<13$ vs subjects without the metabolic syndrome and subjects with a FINDRISC $\geq 13$ vs subjects with the metabolic syndrome

\begin{tabular}{|c|c|c|c|c|c|c|}
\hline & FINDRISC $<13$ & Metabolic healthy & P1 & FINDRISC $\geq 13$ & Metabolic syndrome & P2 \\
\hline Number of cases & 389 & 339 & & 262 & 312 & \\
\hline Age (years) & $40 \pm 12$ & $41 \pm 13$ & 0.89 & $47 \pm 12$ & $45 \pm 12$ & 0.83 \\
\hline Weight (kg) & $108.3 \pm 22.0$ & $104.7 \pm 20.3$ & 0.32 & $110.9 \pm 19.9$ & $114 \pm 20.9$ & 0.02 \\
\hline BMI $\left(\mathrm{kg} / \mathrm{m}^{2}\right)$ & $37.8 \pm 6.2$ & $37.5 \pm 6.2$ & 0.91 & $38.9 \pm 5.8$ & $39.0 \pm 5.8$ & 0.85 \\
\hline Waist (cm) & $113.9 \pm 14.9$ & $113.8 \pm 15.6$ & 0.98 & $119.4 \pm 13.3$ & $120.8 \pm 13.2$ & 0.88 \\
\hline Systolic BP (mmHg) & $126 \pm 15$ & $123 \pm 13$ & 0.76 & $132 \pm 14$ & $134 \pm 14$ & 0.82 \\
\hline Diastolic BP (mmHg) & $75 \pm 11$ & $73 \pm 10$ & 0.89 & $78 \pm 11$ & $80 \pm 11$ & 0.79 \\
\hline Triglycerides (mg/dl) & $125(90-177)$ & $106(80-130)$ & $<0.001$ & $150(108-198)$ & $179(150-1229)$ & 0.01 \\
\hline Cholesterol (mg/dl) & $196(175-226)$ & $197(172-219)$ & 0.86 & $202(173-229)$ & $201(175-235)$ & 0.87 \\
\hline HDL cholesterol (mg/dl) & $48(40-60)$ & $56(48-66)$ & $<0.001$ & $49(40-60)$ & $42(40-60)$ & 0.05 \\
\hline CT visceral fat $\left(\mathrm{cm}^{2}\right)$ & $169(115-225)$ & $155(102-212)$ & $<0.001$ & $206(148-277)$ & $210(158-267)$ & 0.20 \\
\hline CT subcutaneous fat $\left(\mathrm{cm}^{2}\right)$ & $592(592)$ & $601(149)$ & 0.10 & $593(138)$ & $584(137)$ & 0.06 \\
\hline Total fat $\left(\mathrm{cm}^{2}\right)$ & $773 \pm 175$ & $771 \pm 183$ & 0.68 & $812 \pm 166$ & $806 \pm 159$ & 0.52 \\
\hline Fasting glucose (mg/dl) & $84(79-91)$ & $83(78-91)$ & 0.84 & $90(83-99)$ & $92(82-101)$ & 0.83 \\
\hline 2-h OGTT glucose (mg/dl) & $133(114-154)$ & $131(113-155)$ & 0.79 & $155(129-186)$ & $152(150-184)$ & 0.68 \\
\hline Hbalc (\%) & $5.4(5.2-5.6)$ & $5.5(5.2-5.6)$ & 0.56 & $5.6(5.4-5.9)$ & $5.6(5.4-5.9)$ & 0.91 \\
\hline $\mathrm{HbA1c}(\mathrm{mmol} / \mathrm{mol})$ & $36(33-38)$ & $36(33-38)$ & 0.97 & $38(36-41)$ & $38(36-40)$ & 0.98 \\
\hline Microalbuminuria ( $\mu \mathrm{g} / \mathrm{min})$ & $8.0(5-11)$ & $11(5-12)$ & 0.43 & $10.0(6-15)$ & $9.0(6-15)$ & 0.66 \\
\hline FINDRISC score & $10 \pm 2$ & $11 \pm 4$ & 0.74 & $16 \pm 3$ & $13 \pm 4$ & 0.03 \\
\hline HOMA-S & $2.9(1.9-4.8)$ & $2.7(1.8-4.2)$ & 0.63 & $3.9(2.5-6.0)$ & $4.2(2.7-6.0)$ & 0.04 \\
\hline HOMA-B (\%) & $252(164-392)$ & $233(152-369)$ & $<0.03$ & $236(147-337)$ & $261(165-385)$ & $<0.01$ \\
\hline Metbalic syndrome & $228 / 161$ & & & $111 / 151$ & & \\
\hline
\end{tabular}

Data are expressed as mean \pm standard deviation or median (range)

$B M I$ body mass index, BP blood pressure, HDL high density lipoprotein, OGTT oral glucose tolerance test, HOMA-S homeostasis model assessment of insulin resistance, HOMA-B homeostasis model assessment of beta-cell function, $P 1 \mathrm{P}$ value for the comparison between FINDRISC $<13$ and Metabolic Healthy, $P 2 \mathrm{P}$ value for the comparison between FINDRISC $\geq 13$ and metabolic syndrome 
to predict type 2 diabetes. The ability of the FINDRISC to identify subjects with type 2 diabetes mellitus in an overweight and obese population has been shown before [14]. The metabolic syndrome showed a relative good ability to identify subjects with type 2 diabetes according to an aROC of 0.72 with a sensitivity of $75 \%$ and specificity of $55 \%$, the positive predictive value was $17 \%$ and the negative predictive value $95 \%$. However, the aROC of the FINDRISC did not differ significantly compared to the aROC of the metabolic syndrome. Since FPG is one of the components that need to be tested for the metabolic syndrome, we investigated whether adding FPG to the FINDRISC questionnaire would improve its discriminatory accuracy. We observed a significant increase in discriminatory accuracy to 0.91 when FPG was added to the original FINDRISC score. When HbA1c was added instead of FPG, it increased to 0.93. Others previously reported that the performance of a diabetes risk score could be improved by adding biochemical markers. Adding lipids and FPG to the Atherosclerosis Risk in Communities (ARIC) study, the aROC increased from 0.71 to $0.80(\mathrm{p}<0.001)[15]$. In the Framingham Offspring Study, the aROC increased from 0.72 to 0.85 when FPG, HDLcholesterol and triglycerides were added to the original risk score [16]. When FPG, triglycerides, HbA1c, HDLcholesterol and liver enzymes were added to the German Diabetes RISK score, the aROC increased from 0.85 to 0.90 (ref: [17]). More sophisticated tests, such as the estimated insulin resistance (HOMA-IR) or estimated insulin secretion (HOMA-B) did not significantly improve the discriminatory accuracy of the Framingham Offspring Study [16]. In contrast, the San Antonio Heart Study model did improve significantly when adding an OGTT, 1-h plasma glucose or HOMA-IR or HOMA-B $[18,19]$.

However, including FPG or HbA1c concentrations into the model introduces a bias, since you introduce parameters, which are used for the diagnosis of diabetes itself, thereby falsely increasing the discriminative power of such a risk score. Moreover, the introduction of parameters, which are used for the diagnosis of diabetes itself, defeats the whole point of why clinicians should use diabetes risk scores. However, the same is true for the metabolic syndrome. Nevertheless, it is worth mentioning that a non-invasive screening tool combined with a relative inexpensive and feasible biochemical maker is a better tool to identify subjects with diabetes than a cluster of five clinical and biochemical makers, which are needed to diagnose the metabolic syndrome.

The prevalence of the metabolic syndrome increased with increasing FINDRISC score. Several cross-sectional studies have assessed the FINDRISC score as a screening tool for the metabolic syndrome [20, 21]. A Greek study evaluated a simplified FINDRISC score to identify subjects with the metabolic syndrome and found an aROC of 0.71 and 0.76 in men and women, respectively [20] Saaristo et al. [21] examined the prevalence of metabolic syndrome in a Finnish population and found an aROC of 0.73 in men and 0.75 in women. The aROC of the FINDRISC score to identify subjects with the metabolic syndrome in this study was lower than those in other cross-sectional studies. In a prospective study, the FINDRISC showed a reasonable ability to rightfully predict if a subject develops the metabolic syndrome, with an aROC of $0.65 \mathrm{ref}$ [22].

This is the first study where the FINDRISC score and the metabolic syndrome are compared to identify subjects with type 2 diabetes mellitus. A major strength of this study is that it consists of 651 well-characterized subjects, and this can be considered as a large cohort. Another strength is that the FINDRISC questionnaire was taken by a health care professional, thereby limiting inaccuracy and increasing reliability.

A relative limitation is the small number of men analyzed in this study. The subjects included in this study were referred by their general practitioner or came at their own initiative and it is well known that women tend to seek help for medical problems more often and earlier than men. Nevertheless, the number of men are in our opinion sufficient enough to generalize these study results. However, validation of these results in other (i.e. larger) cohorts is desirable. Another limitation is the cross-sectional study nature of this study, precluding causality. It would be interesting to organize follow-up to improve the prediction model, not only for diabetes status but also cardiovascular outcome. Data collection continues as we report.

\section{Conclusion}

In a population consisting of only subjects with overweight or obesity, 72 subjects were newly diagnosed with type 2 diabetes mellitus and 328 were diagnosed with prediabetes. In total, 312 subjects met the diagnostic criteria for the metabolic syndrome. Of these 312 subjects, 151 had a FINDRISC score $>13$ using the NCEP-ATP III criteria.

The discriminatory accuracy to identify subjects with diabetes of the original FINDRISC questionnaire was similar to that of the metabolic syndrome, but much easier to perform since it does not require invasive testing. However, when adding Hba1c or FPG the FINDRISC score outperformed the metabolic syndrome. Therefore, our findings suggest, that it may be of good clinical practice to use the FINDRISC score + HbA1c (with the official cutoff point for diabetes) in a two-step screening 
model for diabetes rather than using the metabolic syndrome.

\section{Abbreviations}

MetS: metabolic syndrome; T2DM: type 2 diabetes mellitus; OGTT: oral glucose tolerance test; BMI: body mass index; HOMA-IR: homeostasis model assessment for insulin resistance; HOMA-B: homeostasis model assessment for beta cell function; NCEP-ATP III: National Cholesterol Education Program Adult Treatment Panel III; FPG: fasting plasma glucose; IFG: impaired fasting glucose; IGT: impaired glucose tolerance; HDL: high-density lipoprotein; aROC: the area under the receiver-operating curve.

\section{Authors' contributions}

All authors made important intellectual contributions to the conception and design of the study. Every author reviewed and provided comments on manuscript drafts and gave final approval of this version to be published. CDB and LVG were responsible for the final design of the protocol. LVG recruited patients and was responsible for the database. All authors implemented the study protocol and acquired data. AM, CDB, IM and AV performed the statistical analysis. AM and CDB drafted the manuscript. All authors read and approved the final manuscript.

\section{Acknowledgements}

We thank the nursing staff and all patients for making this observational study possible.

\section{Competing interests}

The authors declare that they have no competing interests.

\section{Availability of data and materials}

The datasets used and analyzed during the current study are not publicly available due to the individual privacy of the patients included in this study, but they are available from the corresponding author upon reasonable request.

\section{Consent for publication}

Not applicable.

\section{Ethics approval and consent to participate}

The study was approved by the Ethics Committee of the Antwerp University Hospital (EC 1/10/32), and conducted in accordance with the principles of the Declaration of Helsinki as revised in 2008. All study participants gave written informed consent.

\section{Funding}

Not applicable.

\section{Publisher's Note}

Springer Nature remains neutral with regard to jurisdictional claims in published maps and institutional affiliations.

Received: 28 September 2017 Accepted: 8 February 2018

Published online: 01 March 2018

\section{References}

1. International Diabetes Federation (IDF). IDF diabetes atlas. 7th ed; 2015 http://www.idf.org/, https://doi.org/10.1289/image.ehp.v119.i03.
2. Lakka H-M, et al. The metabolic syndrome and total and cardiovascular disease mortality in middle-aged men. JAMA. 2002;288:2709-16.

3. Ford ES, Giles WH, Dietz WH. Prevalence of the metabolic syndrome among US adults. JAMA. 2002;287:356

4. Stern MP, Williams K, González-Villalpando C, Hunt KJ, Haffner SM. Does the metabolic syndrome improve identification of individuals at risk of type 2 diabetes and/or cardiovascular disease? Diabetes Care. 2004;27:2676-81

5. Lorenzo C, et al. The metabolic syndrome as predictor of type 2 diabetes: the San Antonio heart study. Diabetes Care. 2003;26:3153-9.

6. Spijkerman AMW, et al. Diabetic patients detected by population-based stepwise screening already have a diabetic cardiovascular risk profile. Diabetes Care. 2002;25:1784-9.

7. Isomaa B, et al. Cardiovascular morbidity and mortality associated with the metabolic syndrome. Diabetes Care. 2001;24:683-9.

8. Expert Panel on Detection. Evaluation, and treatment of high blood cholesterol in adults. Executive summary of the third report of the National Cholesterol Education Program (NCEP) expert panel on detection, evaluation, and treatment of high blood cholesterol in adults (Adult Treatment Panel III). JAMA. 2001:285:2486-97.

9. Saaristo T, et al. National type 2 diabetes prevention programme in Finland: FIN-D2D. Int J Circumpolar Health. 2007:66:101-12.

10. van der Kooy K, Seidell JC. Techniques for the measurement of visceral fat: a practical guide. Int J Obes Relat Metab Disord. 1993;17:187-96.

11. Kvist H, Chowdhury B, Grangard U, Tylen U, Sjostrom L. Total and visceral adipose-tissue volumes derived from measurements with computed tomography in adult men and women: predictive equations. Am J Clin Nutr. 1988;48:1351-61.

12. Matthews DR, Hosker JP, Rudenski AS, Naylor BA, Treacher DF. T. R. Homeostasis model assessment insulin resistance and beta-cell function from fasting plasma glucose and insulin concentrations in man. Diabetologia. 1985;28:412-9.

13. ADA. Classification and diagnosis of diabetes. Diabetes Care. 2016;39:13-22

14. Meijnikman AS, et al. Screening for type 2 diabetes mellitus in overweight and obese subjects made easy by the FINDRISC score. J Diabetes Complicat. 2016;30:1043-9.

15. Schmidt Ml, et al. Identifying individuals at high risk for diabetes: the atherosclerosis risk in communities study. Diabetes Care. 2005;28:2013-8.

16. Wilson PWF, et al. Prediction of incident diabetes mellitus in middle-aged adults. Arch Intern Med. 2007;167:1068.

17. Schulze MB, et al. Use of multiple metabolic and genetic markers to improve the prediction of type 2 diabetes: the EPIC-Potsdam Study. Diabetes Care 2009:32:2116-9.

18. Abdul-Ghani MA, Williams K, DeFronzo RA, Stern M. What is the best predictor of future type 2 diabetes? Diabetes Care. 2007:30:1544-8.

19. Stern MP, Williams K, Haffner SM. Identification of persons at high risk for type 2 diabetes mellitus: do we need the oral glucose tolerance test? Ann Intern Med. 2002;136:575-81.

20. Makrilakis K, et al. Validation of the Finnish Diabetes Risk Score (FINDRISC) questionnaire for screening for undiagnosed type 2 diabetes, dysglycaemia and the metabolic syndrome in Greece. Diabetes Metab. 2011;37:144-51.

21. Saaristo T, et al. Cross-sectional evaluation of the Finnish diabetes risk score: a tool to identify undetected type 2 diabetes, abnormal glucose tolerance and metabolic syndrome. Diabetes Vasc Dis Res. 2005;2:67-72.

22. Janghorbani $M$, Adineh $H$, Amini M. Evaluation of the Finnish Diabetes Risk Score (FINDRISC) as a screening tool for the metabolic syndrome. Rev Diabet Stud. 2013;10:283-92. 